\title{
LES DEUX CHRISTIANISMES DE LOCKE ET DE TOLAND
}

\author{
Geneviève BRYKMAN
}

\begin{abstract}
RÉSUMÉ: La proximité chronologique et conceptuelle de Reasonableness of Christianity (1695) avec le Christianity not Mysterious (1696) de Toland eut tôt fait d'être, pour Locke, tout à fait compromettante : Toland semblait s'appuyer sur la théorie de la connaissance de l'Essai sur l'entendement humain, pour produire son interprétation incendiaire des Écritures. Faire de Locke un précurseur de Toland serait pourtant une abusive simplification. En s'appuyant sur le livre IV de l'Essai, Reasonableness of Christianity, le Discourse on Miracles et sur A Paraphrase and Notes on St Paul's Epistles, pour Locke et, pour Toland, sur Christianity not Mysterious, les Letters to Serena (1704), et le Nazarenus (1718), on peut montrer qu'il existe une véritable discontinuité entre deux représentations divergentes du christianisme.
\end{abstract}

Mots clés : raison, révélation, mystère, herméneutique.

SUMMARY : The chronological and conceptual affinity of Reasonableness of Christianity (1695) with Christianity not Mysterious (1696) was before long considered as a compromising evidence of Locke's influence : Toland was straight away perceived as if the theory of knowledge included in the Essay was a genuine source of his subversive interpretation of Christianity. However, to think of Locke as a precursor of Toland would be an over-simplification. Relying mainly on Book IV of the Essay (1690), Reasonableness of Christianity, A Discourse of Miracles, and A Paraphrase and Notes on St Paul's Epistles, on one hand; on Christianity not Mysterious, Letters to Serena (1704), and Nazarenus (1718), on the other, we will set forth a basic disparity between Locke and Toland regarding their divergent views on Christianity.

Keywords : reason, revelation, mystery, hermeneutics. 
Zusammenfassung : Die chronologische und gedankliche Nähe von Reasonableness of Christianity (1695) und Christianity not Mysterious (1696) von Toland wurde bald für Locke ganz und gar kompromittierend. Tatsächlich schien sich Toland bei seiner aufhetzerischen Interpretation der Heiligen Schrift auf die Erkenntnistheorie des Essai zu berufen. Aber aus Locke einen Vorläufer von Toland zu machen wäre eine übertriebene Vereinfachung. Wenn man sich auf das Buch IV des Essai, Reasonableness of Christianity, Discourse on Miracles und auf A Paraphrase and Notes on St Paul's Epistles, was Locke betrifft und was Toland betrifft, auf Christianity not Mysterious, Letters to Serena (1704) und Nazarenus (1718) bezieht, kann man zeigen, daß es eine echte Diskontinuität zwischen zwei divergierenden Auffassungen von Christentum gibt.

Stichwörter : Verstand, Offenbarung, Mysterium, Hermeneutik.

Geneviève BrykmaN, née en 1941, est ancienne élève de l'École normale supérieure de Fontenay. Agrégée de philosophie, docteur ès lettres, elle est professeur à l'université Paris XNanterre. Ses recherches portent principalement sur Berkeley, la philosophie anglaise classique et la diffusion du spinozisme en Angleterre au seuil du xvw' siècle.

Adresse: U.F.R.-L.L.Phi, Université de Paris X-Nanterre, 200 av. de la République, 92001 Nanterre Cedex. 
D'entrée, une ambiguiité du titre de cet exposé mérite d'être levée, parfois entretenue, aussi bien par la réception immédiate des ouvrages de Locke et de Toland*, que par certains commentaires contemporains ${ }^{1}$ : il ne s'agit pas ici de traiter de deux christianismes de Locke, puis de deux christianismes de Toland, dont, dans les deux cas, l'un serait une religion à l'usage du vulgaire et l'autre un christianisme épuré, proche du déisme; il s'agit, sans préjuger de leur éventuel «double langage», de comparer la conception que Locke et Toland se font respectivement du christianisme.

Il est vrai que la proximité chronologique et conceptuelle de Reasonableness of Christianity (1695) avec le Christianity not Mysterious (1696) de Toland eut tôt fait d'être, pour Locke, tout à fait compromettante : Toland semblait s'appuyer sur la théorie de la connaissance de l'Essai, pour produire son interprétation incendiaire des Écritures ${ }^{2}$. Comme le titre même de l'ouvrage, Reasonableness of Christianity, semblait faire trop belle part à la raison humaine, on a pu croire que Locke et Toland se trouvaient dans une même famille subversive; tout au plus Locke aurait été, ou plus timide, ou plus prudent que Toland, dans sa rationalisation des « choses divines »; mais son Essai aurait déroulé un tapis rouge pour le christianisme de Toland.

Faire de Locke un précurseur de Toland est pourtant une abusive simplification. En nous appuyant sur l'Essai (livre IV, 1690), Reasonableness of Christianity, le Discourse on Miracles (1701) et sur les Notes on St Paul's Epistles (1702), d'une part, et, d'autre part, sur Christianity not Mysterious, les Letters to Serena (1704) et sur le Nazarenus (1718), nous montrerons qu'il y a solution de continuité entre la représentation du christianisme par Locke et celle que propose Toland : coupure dans la conception du contenu de la croyance religieuse, mais aussi rupture correspondante dans la conception de l'exégèse des Écritures. En sorte que le christianisme de Locke et celui de Toland pourraient bien être, non seulement différents, mais antinomiques.

* Les cuvres de Locke sont citées dans l'édition suivante: The Works of John Locke, 9 vol., $9^{e}$ éd., Londres, 1794. Les cuvres de Toland sont présentées une à une en note.

1. Herbert J. McLachlan, Socinianism in the 17th Century England, Oxford, 1951, p. 351 sq.; Margaret C. JACOB, «John Toland and Newtonian Ideology », Journal of the Warburg and Courtauld Institute, 32, 1969 ; David BERMAN, « Deism, Immortality and the Art of Theological Lying ", in Deism, Masonry and the Enlightenment, Newark, N.J./Londres/Toronto, University of Delaware Press, 1987, et A History of Atheism in England, Oxford, 1988.

2. John Toland, Christianity not Mysterious (cité par la suite comme CNM), FaksimileNeudruck der Erstausgabe, Londres, 1696, Stuttgart, Frommann, 1964, sect. I, chap. II sect. II, chap. I. 
L'intérêt de Locke pour le commentaire des Écritures n'est pas le repli sur soi d'un homme vieillissant ${ }^{3}$. Il est suffisamment attesté que l'herméneutique biblique ne cessa de constituer un pôle de réflexion tout au long de l'itinéraire intellectuel du philosophe. Locke lui-même, dans l'Essai, ne manque pas de faire apparaître la solidarité entre l'établissement d'une philosophie de la connaissance et l'intérêt incontournable pour les objectifs pratiques des hommes ${ }^{4}$. Si donc l'attention pour la croyance religieuse semble un trait objectif dominant des derniers écrits, ceux-ci ne manquent pas d'obéir au souci de compléter l'enquête menée dans l'Essai sur l'entendement humain par une enquête plus directe sur les sphères respectives de la raison et de la foi.

Sur la raison et la foi, le quatrième livre de l'Essai semblait déjà dessiner les contours d'une « religion rationnelle », qui préparait au reste fort bien le terrain pour les accusations de socinianisme ${ }^{5}$. Ayant souligné l'extrême limitation de la connaissance certaine (knowledge) que les hommes obtiennent dans la lumière de l'intuition (intuition par laquelle on perçoit immédiatement l'accord ou le désaccord entre deux idées par exemple, que blanc n'est pas noir, ou que 2 est plus grand que 1$)^{6}$, Locke accordait la plus grande place à la connaissance probable, avec tous les degrés qu'imposait le recours à des idées intermédiaires ${ }^{7}$ et l'absence de perception claire de leurs liaisons. Pour vivre et pour connaître, Dieu ne nous a pas donné ici-bas la faveur d'une évidence rationnelle permanente, mais seulement la lumière crépusculaire du probable ${ }^{8}$, plus adaptée à la médiocrité de notre condition. Or cette place accordée à la connaissance probable, avec tous ses degrés, faisait du jugement (judgment) un mode de connaissance privilégié qui recouvre tous les assentiments de l'esprit à des objets de connaissance dont la vérité est seulement présumée 9 . Dès lors, pour une épistémologie de la croyance religieuse, la question de la véracité des témoignages humains et divins

3. Maria Christina PrTassı, «Le philosophe et l'Écriture, John Locke exégète de saint Paul ", Cahiers de la Revue de théologie et de philosophie, 1990, p. 16.

4. Essay, IV, chap. XIv, sect. 1: «Man would be at a great loss if he had nothing to direct him [for the conduct of his life], but what has the certainty of true knowledge. For that being very short and scanty, as we have seen, he would be often utterly in the dark, and in most of the actions of his life perfectly at stand, had he nothing to guide him in the absence of clear and certain knowledge."

5. Edward Strlingfleet, A Discourse in Vindication of the Doctrine of the Trinity, 1697, attaquait directement le Christianity not Mysterious de Toland, en y associant les présupposés fournis par Locke dans l'Essai; John EDwards, Some Thoughts Concerning the Several Causes and Occasions of Atheism, 1695; Socinianism Unmasked, 1696.

6. Essay, IV, chap. II : "Of the Degrees of our Knowledge », sect. 1.

7. Essay, IV, chap. II, sect. 2.

8. Essay, IV, chap. xIV, sect. 2.

9. Essay, IV, chap. XIV, sect. 4. 
devient centrale. L'analyse de la notion de miracle prend, du même coup, une importance décisive, tandis que la notion de mystère est laissée dans une pénombre qu'il nous faudra interroger. À l'inverse, on verra que, chez Toland, l'examen critique des " mystères » de la religion chrétienne officielle, rend la question de la nature des miracles ouvertement marginale ${ }^{10}$. Si l'on a pu parler de religion rationnelle chez Locke ${ }^{11}$, c'est que, pour l'auteur de l'Essai, c'est à la raison qu'il revient de faire une critique des témoignages, critique qui permet de découvrir certains prétendus envoyés de Dieu comme des imposteurs. Toutefois, c'est la définition même de la raison qui est à la source des divergences entre Locke et Toland, car elle a pour enjeux le contenu même du christianisme et la conception de l'exégèse des Écritures.

Dans l'Essai, Locke maintient que la connaissance que nous avons de quelque chose comme étant une révélation d'origine divine n'est jamais aussi certaine que la connaissance que nous obtenons par nous-mêmes, soit par les voies immédiates de la sensation et de la réflexion, soit par la voie de la déduction rationnelle. Ainsi, nul homme inspiré de Dieu ne peut communiquer aux autres hommes une idée simple radicalement nouvelle, dont ils n'auraient eu auparavant la moindre expérience. Selon Locke, un tel homme doit, en quelque sorte, être considéré comme doué d'un sixième sens : il se trouve dans la situation d'un être humain doué de la vue, et qui chercherait à décrire les diverses couleurs à un aveugle-né. C'est ainsi qu'on peut comprendre la description selon laquelle saint Paul, revenu du «troisième ciel », se trouva dans la difficulté d'exprimer à ses congénères « toutes ces choses que l'œil n'a point vues, que l'oreille n'a point entendues, et qu'il n'a jamais été accordé aux hommes de concevoir ». D'autre part, Locke observe que les êtres humains pourraient, sur la foi de la tradition, consentir à la vérité de la proposition que « les trois angles d'un triangle sont égaux à deux droits », s'il avait été reconnu depuis plusieurs siècles que cette proposition était une révélation de Dieu. Mais, précise-t-il, une telle révélation n'arriverait jamais à un si haut degré de certitude que celle que je peux obtenir en comparant et mesurant mes propres idées ${ }^{12}$.

10. CNM, sect. III, chap. Iv, $\S 69-74:$ « When all other shifts prove ineffectual, the partisans of mysteries fly to miracles as their last refuge; but this is to weak a place to make any long resistance, and we doubt not of beating them quickly thence with ease and safety. »Outre SPINOZA, in Traité des autorités théologiques et politiques (cité par la suite comme TTP), 1665 , chap. vi, Toland avait à sa disposition la critique des miracles de Charles BlouNT, Miracles, No Violations of the Laws of Nature, 1683, elle-même largement inspirée du TTP.

11. Richard S. WeSTFALL, Science and Religion in Seventeenth-Century England, Newhaven, 1958, chap. 5 et 7 ; Massimo FirPo, « John Locke e il socinianesimo », Rivista storica italiana, XCII, 1980 , p. 35-124.

12. Essay, IV, chap. xviII, sect. 3. 
Cependant, Locke souligne que, pour nous avoir accordé la lumière de la raison avec parcimonie, Dieu s'est réservé la liberté de nous apporter le secours de la révélation sur les matières qui, pour nos seules facultés naturelles, demeureraient probables ${ }^{13}$; dans ce cas, la révélation doit l'emporter sur les conjectures probables de la raison. Y a-t-il dès lors dévaluation de la raison? Selon Locke, point du tout; car il appartient toujours à la raison : 1) de trancher la question cruciale de savoir si c'est bien d'une révélation divine qu'il s'agit; 2) de déterminer quel est le sens des paroles en lesquelles cette révélation est proposée ${ }^{14}$. En sorte que, en un rationalisme qui semble rigoureux et non pas seulement « raisonnable ", l'Essai affirme tout à la fois qu'on ne saurait douter de ce que Dieu a révélé (car Dieu ne peut pas mentir), et que, pour savoir s'il s'agit bien d'une révélation divine, il faut se tenir à ce principe que nulle chose contraire à, ou incompatible avec la raison ne peut être objet de foi ${ }^{15}$.

Le principe ci-dessus ne doit pourtant pas faire illusion quant à la rigidité du rationalisme de Locke. C'est la définition même de la raison qui fait problème, dès lors que, à l'encontre de ce que laisse attendre le titre du chapitre XVIII, « De la foi et de la raison, et de leurs bornes distinctes », Locke gomme quelque peu la distinction entre foi et raison lorsqu'il déclare que :

"La raison est une révélation naturelle, par où [Dieu] communique aux hommes cette portion de vérité qu'il a mise à la portée de leurs facultés naturelles; et la révélation est la raison naturelle, augmentée par un fond nouveau de découvertes émanées immédiatement de Dieu » ${ }^{16}$.

Le chapitre consacré à la raison en proposait déjà une description qu'on peut dire libérale : cette faculté serait selon Locke, vouée à la considération des idées intermédiaires (les «idées moyennes", dira Coste) et non plus aux natures simples, principes ou axiomes innés d'autres philosophies. Constatant que l'expérience sensible et l'intuition ne nous permettent pas de progresser très avant dans la connaissance, Locke tient que c'est à la raison qu'il revient de découvrir des idées moyennes, et de les enchaîner correctement. Or, découvrir et appliquer de telles idées est, selon lui, le propre des deux facultés qu'il accorde à la raison humaine : la sagacité, ou faculté de découvrir de bons principes, et la faculté d'inférer. Et comme la plus grande partie de notre connaissance dépend des idées moyennes, nous sommes conduits à substituer l'assentiment au savoir, et à

13. Essay, IV, chap. xviI, sect. 8 .

14. Essay, ibid.

15. Essay, IV, chap. xVIII, sect. 10.

16. Essay, IV, chap. XIx, "On Enthusiasm », sect. 4. 
tenir pour vraies certaines propositions, sans avoir à leur sujet de certitude véritable. Cependant, il nous faut examiner et comparer les fondements de leur probabilité; et c'est là la fonction principale de la raison, dont Locke propose la définition nominale suivante :

«La faculté qui découvre et applique comme il faut les moyens nécessaires pour trouver la certitude dans l'une (la découverte des idées moyennes) ou la probabilité dans l'autre (l'enchaînement de nos idées) est ce que nous appelons raison $" 17$.

Cette définition, qui intègre d'emblée le probable, décrit une raison raisonnable ${ }^{18}$, plutôt que strictement rationnelle; et elle prépare très directement le terrain pour Reasonableness of Christianity.

En effet, dans ce contexte conceptuel, Locke accepte de dire que, si aucune vérité ne peut contredire la raison, il demeure cependant des vérités qui sont au-dessus de la raison, à savoir, « des propositions dont nous ne voyons pas que la vérité ou la probabilité puisse être déduite par le seul secours de la raison ${ }^{19}$. Il y a donc quelque chose comme des mystères; et l'Essai admet explicitement tout un espace que la pénombre de la raison humaine ne saurait investir.

Sur ce chef de la raison, il nous faut reprendre l'excellente formule de P. Lurbe :

« $\grave{A}$ lire Toland après Locke, on a le sentiment de retrouver un dogmatisme abrupt; on se meut dans un monde rigoureusement binaire, où n'existent que les seules catégories du vrai et du faux $»{ }^{20}$.

Dans Christianity not Mysterious, en effet, en dépit des apparences créées par des catégories conceptuelles souvent empruntées à Locke, Toland propose une définition tout autre de la raison, qui rapporte immédiatement celle-ci à l'évidence d'idées simples et distinctes ${ }^{21}$. Dès le début de l'ouvrage, Toland appelle raison:

« la faculté de l'âme qui découvre la certitude de quelque chose de douteux ou d'obscur, en le comparant à quelque chose de connu dans l'évidence ${ }^{22}$.

17. Essay, IV, chap. xVII, sect. 2.

18. L'expression est de Simone GoYARD-FABRE, John Locke et la raison raisonnable, Paris, Vrin, 1986.

19. Essay, IV, chap. XVI, sect. 23. Pour éclairer les distinctions devenues traditionnelles, Locke donne les exemples suivants : « the existence of one God is according to reason; the existence of more than one God, contrary to reason; the resurrection of the dead, above reason. »

20. Pierre LuRbe, «Le spinozisme de John Toland », in Spinoza au xviff siècle, éd. par Olivier Bloch, Paris, Klincksieck, 1990, p. 39.

21. $C N M$, sect. I, chap. II, $\$ 5$.

22. CNM, sect. I, chap. II, $\$ 7$. 
Qu'est-ce encore que cette évidence? Alors que pour Locke, celle-ci admet des degrés, correspondant à tous les degrés de la connaissance probable, Toland répond que l'évidence se tient dans l' "exacte conformité " de nos idées ou pensées avec leurs objets ${ }^{23}$. Il ne paraît pas ici se préoccuper du critère relatif à cette « conformité », mais il donne, par ailleurs, de la puissance du vrai à être reconnu par lui-même, une description toute spinoziste dont il se sert pour identifier vérité et révélation ${ }^{24}$.

À partir du point d'ancrage que lui fournit l'évidence comme expérience sui generis, Toland procède à un balayage radical des mystères et se donnera tout au long pour tâche de montrer : 1) que rien, dans l'Évangile, ne peut être contraire à la raison; 2) qu'il n'y a rien non plus qu'on puisse y reconnaître comme au-dessus de la raison, sans s'exposer à toutes sortes d'absurdités et à de multiples dangers pour la vie sociale ${ }^{25}$.

La conception de la raison chez Locke et Toland est la matrice de bien d'autres différences. Ainsi en est-il de la représentation du mystère; ainsi en est-il de la représentation de l'exégèse des Écritures; et ainsi en sera-t-il de la conception de la fonction sociale du phénomène religieux.

Dans Reasonableness of Christianity, Locke se propose d'établir que le christianisme est une religion raisonnable en ce sens qu'on peut la réduire à une croyance naturelle des plus simples. En d'autres termes, il s'agit de ramener entièrement le christianisme à la seule doctrine de la « justification par la foi ». Et cette doctrine se dédouble, d'une part, en la croyance unique selon laquelle Jésus-Christ est le Messie; d'autre part, en l'obligation de vivre selon la morale enseignée par Jésus.

Il y avait là, certes, de quoi inquiéter les théologiens et le clergé anglican, car Locke décrit un christianisme minimaliste, qu'on peut dire latitudinaire : il considère que la religion chrétienne n'implique, ni le dogme du péché originel, ni la doctrine du rachat qui lui est traditionnellement associée. Selon Locke, la « vraie religion » n'exige que la croyance toute simple selon laquelle le Christ s'est « levé d'entre les morts » et a apporté l'assurance de l'immortalité à ceux qui vivraient selon « la loi de la foi ». Selon cette loi, chacun n'est responsable que devant Dieu, et ne l'est que pour ses propres péchés; l'intention individuelle de bien faire compte plus que les actions elles-mêmes. La loi mosaïque aurait été seulement, selon Locke, une « loi des œuvres », adaptée à l'État des Hébreux, et que le message du Christ dépassait dans un enseignement de portée univer-

23. CNM, sect. I, chap. IV, $\S 12$ : « Evidence consists in the exact conformity of our ideas or thoughts with their objects, or the things we think upon. »

24. CNM, sect. I, chap. In, \& 11; voir infra n. 42 .

25. CNM, sect. II, chap. I; sect. III, chap. I. 
selle $^{26}$. Il n'y a cependant pas d'opposition entre la loi de Moïse, déjà conforme à la « loi de nature », et l'enseignement du Christ. Le caractère « raisonnable » du christianisme vient précisément de sa capacité d'intégration.

On notera que la « loi de la foi » accorde, comme l'Essai lui-même, la plus grande place à la conscience individuelle, radicalement privée, d'un sujet que Locke décrit comme seul avec Dieu; la religion n'étant, qu'après coup, une communion volontaire de croyants ${ }^{27}$. S'appuyant sur cette conception de la conscience ${ }^{28}$, le Discourse on Miracles s'ouvrait sur une définition subjectiviste du miracle :

" Une action sensible qui, étant au-dessus de la compréhension du spectateur et, de son point de vue, contraire au cours établi de la nature, est considérée par lui comme divine ${ }^{29}$.

D'une telle définition, il suit que ce qui peut être tenu pour extraordinaire par les uns, peut ne pas l'être par d'autres. Reste donc à savoir comment certains phénomènes pourront être tenus pour de véritables miracles. Aussi Locke s'attache-t-il longuement à proposer des critères « raisonnables » pour cette reconnaissance : la puissance surnaturelle manifestée par certaines actions comparées à d'autres ${ }^{30}$, le nombre et la variété des miracles exécutés par une seule personne ${ }^{31}$, donnent à croire qu'aucune révélation ne peut rivaliser avec celle qui vient authentiquement de Dieu. Entreprise périlleuse, voire circulaire, fera remarquer Toland, car il faut déjà croire en Dieu pour croire que le miracle divin est possible ${ }^{32}$.

Dans l'Essai, la « vraie religion » était à peine évoquée en tant que telle (et ne l'était que par le biais des réflexions de Locke sur la foi).

26. Les analogies avec le TTP de Spinoza, quoique plus discrètes que chez Toland, sont ici bien réelles. L'intérêt de l'auteur pour les ouvrages de Spinoza est attesté par l'acquisition, en 1683, de l'édition des Opera Posthuma, ainsi que par la présence de plusieurs éditions du $T T P$, dans la bibliothèque de Locke. Sur ce point, voir : John Harrison et Peter LasLetT, The Library of John Locke, Oxford, Oxford Bibliographical Society Publications, n. s. 13, 1965, $2^{\mathrm{e}}$ éd., 1971.

27. A Letter on Toleration (cité par la suite comme $L T$ ), Works, V, p. 13.

28. Voir Gary WEDEKING, "Locke on Personal Identity and the Trinity Controversy of the 1690s », Dialogue, vol. XXIX, 2, 1990.

29. Discourse on Miracles (cité par la suite comme DM), Works, VIII, p. 256.

30. Ibid. : "The producing of serpents, blood, and frogs by the Egyptians sorcerers and by Moses, could not to the spectators but appear equally miraculous [...]. But, when Moses'serpent eat up theirs, when he produced lice, which they could not, the decision was easy. 》

31. Ibid. : «So likewise, the number, variety and greatness of the miracles wrought for the confirmation of the doctrine delivered by Jesus-Christ. 》

32. CNM, sect. II, chap. I, \& 5-6, p. 31. Sur cette difficulté, voir Richard E. AsHCRafT, « Faith and Knowledge in Locke's Philosophy », in John Locke, Problems and Perspectives, Cambridge, John W. Yolton, 1969. 
Dans Reasonableness of Christianity, elle l'est constamment et est identifiée à «l'Église du Christ». Locke précise que cette religion, bien qu'elle concerne tous les hommes, est particulièrement adaptée à ceux qui ne peuvent pas penser par eux-mêmes ${ }^{33}$. Aussi, le christianisme de Locke est-il un christianisme moral, où l'obéissance à la règle de justice et de piété est plus importante que la connaissance des attributs de Dieu.

C'est pourquoi, sans doute, le philosophe se montre très réservé quant à dire explicitement que Jésus est le « fils de Dieu »: il tend à présenter cette expression comme une concession de Jésus à ceux qui l'accusent de semer la sédition en se prétendant "roi des Juifs » ${ }^{34}$. Et Locke est plus discret encore quant à déclarer que le mystère de la Trinité est un contenu indispensable de la religion chrétienne ${ }^{35}$; il pourrait bien s'agir d'un dogme, issu des spéculations téméraires de théologiens, sur des thèmes qui ne sont nullement dans la Bible. Cependant, c'est bien un mystère qui demeure la pierre angulaire du christianisme de Locke: le mystère de la messianité du Christ, un messager de Dieu que Locke ne met pas sur la même ligne que Moïse ou les prophètes. Et le miracle de la résurrection du Messie semble rétrospectivement donner, à d'autres actions du Christ, leur caractère divin.

Au regard de l'attitude à la fois critique et très mesurée de Locke, on aborde, avec les ouvrages de Toland, un véritable combat contre l'obscurantisme et contre le pouvoir exorbitant des prêtres ${ }^{36}$. Loin de faire de Toland un éclectique, ce combat traverse son ouvre comme une lame de fond et lui donne une indiscutable cohérence. Mais il fait passer l'épistémologie de la croyance religieuse au second $\operatorname{plan}^{37}$ : là où Locke faisait œuvre de philosophe, soucieux d'établir les conditions originaires du savoir, Toland déclare explicitement, dans Le Nazaréen ${ }^{38}$, se livrer à un

33. Reasonableness of Christianity (cité par la suite comme RC), Works, VI, p. 146 : «The greatest part of mankind want leisure or capacity for demonstration; nor can carry a train of proofs [...]. The greatest part cannot know, and therefore they must believe. " Voir aussi ibid., p. $157-158$.

34. Ibid., p. 83-84. Locke met cette affirmation dans la bouche de saint Pierre, mais sans insistance : "When he asked them, who they thought him to be, and Peter answered, "The Messiah, the Son of God", Matt. XVI, 16, he plainly shows by the following words, that he himself had not told them so."

35. Locke's Second Reply to Stillingfleet, Works, IV, p. 341.

36. Justin ChAMPION, The Pillars of Priestcraft Shaken: The Church of England and its Enemies (1660-1730), Cambridge/New York, Cambridge University Press, 1992.

37. P. LuRBE, art. cit. supra n. 20.

38. Cet ouvrage Nazarenus sera cité ci-après dans l'édition française publiée à Londres en 1777 (cité par la suite comme Le Nazaréen); il comprend de fait deux « Lettres » : la première étant $L e$ Nazaréen stricto sensu et la seconde une Dissertation sur un manuscrit irlandais relatif à l'Évangile de Barnabé. 
travail d' « historien ${ }^{39}$ (la question de sa partialité restant ouverte). Il accorde une très grande place à l'accumulation et à la critique des documents, ainsi qu'à la convergence des témoignages, dont il fait l'indice d'existence de «notions communes » parmi les hommes.

Le Christianity not Mysterious se donnait, il est vrai, une allure philosophique par ses questions initiales : qu'est-ce que la raison? qu'est-ce que l'évidence? Mais de telles questions étaient toutes organisées en une réponse lapidaire, qui nous met d'emblée fort à distance du christianisme de Locke.

Il n'y a rien, dans l'Écriture, qui soit contraire à la raison ou au-dessus de la raison; en bref, rien qui ne soit parfaitement intelligible ${ }^{40}$. Qu'il n'y ait rien qui soit contraire à la raison, chacun peut le vérifier en faisant appel à ce sens commun dont disposent également tous les êtres humains (hommes, femmes, ou même enfants), avant que l'éducation ne les ait rendus esclaves de leurs préjugés ${ }^{41}$. Qu'il n'y ait rien qui soit au-dessus de la raison se découvre dès que l'on s'arrête avec quelque attention sur le sens des mots « mystère » et « révélation ». Selon Toland, toute révélation doit s'entendre en un sens propre et correspond à ce qu'il détermine comme un moyen d'information ${ }^{42}$ : révéler, c'est faire connaître ce qui, connu de certains, était ignoré par d'autres. Et Toland distingue encore ici : 1) l'autorité humaine, qu'on appelle parfois «certitude morale »; certitude qu'on accorde, par exemple, à l'existence d'un pays où nous n'avons jamais mis les pieds, mais où d'autres sont allés et en rapportent des souvenirs ${ }^{43}$; 2) l'autorité divine ou révélation divine, que Toland définit comme "la manifestation de la vérité par elle-même $»^{44}$. Qu'autorité humaine et autorité divine soient aussi différentes que la connaissance par ouï-dire et la

39. Le Nazaréen, chap. I, p. 10 : «Quoiqu'en général je n'ai prétendu que faire un travail d'historien, et que je ne me sois permis de faire de réflexions que celles qui conviendront à mon sujet que j'ai puisé dans la Bible et dans les Pères, je ne laisserai pas, quand il est besoin, d'indiquer des méthodes, par lesquelles on pourra raisonnablement réfuter les erreurs des ignorans ou des méchans. »

40. CNM, sect. II, Introd. Ayant auparavant distingué les moyens d'information, du fondement de la persuasion, Toland poursuit : "I take it to be very intelligible from the precedent section, that what is evidently repugnant to clear and distinct ideas, or to our common notions, is contrary to reason; I go on to prove that the doctrines of the Gospel, if it be the word of God, cannot be so."

41. Letters to Serena, Faksimile-Neudruck der Ausgabe, Londres, 1704, Stuttgart, Frommann, 1964, Letter I, «The Force and Origin of Prejudices ».

42. CNM, sect. I, chap. III, p. 10-11: « Authority, abusively so called, as if all its informations were to be received without examen, is either humane or divine: humane authority is call'd also moral certitude [...] The authority of God, or divine revelation, is the manifestation of truth by truth itself. " On notera la proximité de cette définition de la révélation avec celle de Spinoza selon laquelle la vérité est à elle-même sa propre marque et celle du faux.

43. CNM, sect. II, chap. II : "Whoever reveals anything, that is, whoever tells us something we did not know before, his words must be intelligible, and the matter possible. This rule holds good, let God or man be the revealer."

44. Voir supra n. 10. 
connaissance par raison, n'empêche pas que, selon Toland, une même rationalité vaille pour l'homme et pour Dieu; c'est une même raison qui, pour l'un et pour l'autre, détermine la manifestation de la vérité par ellemême. L'omnipotence de Dieu, concernant la révélation de faits passés ou futurs, est limitée par ce qui est rationnellement possible ${ }^{45}$, mais cette condition limitative permet précisément à l'être humain de reconnaître la vérité. Et c'est à partir de cette limitation même que Toland met en cause l'existence de mystères dans le christianisme.

Ayant montré que rien de contraire à la raison ne peut être objet de foi ${ }^{46}$, Toland distingue deux sens du mot «mystère »: ou bien ce mot désigne quelque chose d'inconcevable pour nos facultés ordinaires; ou bien il désigne quelque chose d'intelligible en soi-même, mais de dissimulé par un rituel compliqué et par un langage figuré ${ }^{47}$. Or, selon Toland, le mot « mystère » n'est pas, dans les Écritures, employé pour désigner une proposition absolument inconcevable, mais seulement pour désigner une doctrine dissimulée par une classe de gens et, dans le même temps, inconnue de l'ensemble de l'humanité ${ }^{48}$; ainsi on comprendra, sans en appeler à une mission surnaturelle du Christ, que ce dernier ait dévoilé, par son enseignement universaliste, ce qui demeurait caché auparavant par ceux qui se flattaient d'être les seuls interprètes de Dieu ${ }^{49}$. En somme, la révélation du Christ est un événement daté, qui rend caduques tous les prétendus mystères qui le précèdent.

Donc, un point de vue « sainement » historique sur le texte du Nouveau Testament en fait un document parmi d'autres. Pas plus dans la religion

45. CNM, sect. II, chap. II : «They impose upon themselves and others, who require assent to things contradictory because God, say they, can do all things, and it were limiting of his power to affirm the contrary. Very good! we heartily believe God can do all things. But that meer nothing should be the object of his power, the very omnipotency alleg'd will not permit us to conceive. "

46. CNM, sect. III, chap. 1 : "That there is nothing Mysterious or above reason in the Gospel » : « This expression [above reason] is taken in a twofold signification. First it denotes a thing intelligible of itself, but so covered by figurative words, types, and ceremonies that reason cannot penetrate the veil, nor see what is under it, till it be removed. Secondly, it is made to signify a thing in its own nature inconceivable and not to be judge by our ordinary faculties and ideas [...]. In the New Testament, "mystery" is always taken in the first sense of the word, viz : for things very intelligible but so covered by figurative words or rites that reason could not discover them without a special revelation; but the veil is actually taken away, then it manifestly follows that the doctrines so revealed cannot now be called properly "mysteries". "

47. CNM, sect. II, chap. I, \& 5-6.

48. Ibid. : «From what has been said it is clear that [the priests] understood by "mystery", in those days, a thing intelligible of itself but so veiled by others that it could not be known without a special revelation. 》

49. Tout le chap. III, de la section III de $C N M$ est destiné à faire la preuve de cette conclusion : "No doctrine then of the Gospel is still a mystery (for the apostles concealed nothing from us that was useful, and have acquainted us with the whole counsel of God); but it is the Gospel itself that was heretofore indeed a mystery, and cannot now after it is fully revealed properly deserve that appellation. " 
que dans les sciences, rien ne peut être dit au-dessus de la raison; et, s'il fallait parler de "mystère » chaque fois que nous n'avons pas d'idée adéquate de quelque chose, alors il y aurait des mystères partout, et tout le langage ordinaire même devrait être tenu pour mystérieux. Avec beaucoup d'habileté tactique, Toland fait observer que, comme Locke l'avait vu en proposant la distinction entre essence réelle et essence nominale, nous ne connaissons pas la structure ultime des choses, et, cependant, nous comprenons fort bien le sens des mots par lesquels nous les désignons, à partir des seules propriétés qui nous sont connues. Si donc nous n'avons aucune idée de quelque chose, c'est perdre notre temps que d'en faire un mystère ${ }^{50}$; et perdre notre liberté que d'accepter d'adorer des entités purement verbales $^{51}$.

Vidé de ses mystères, le christianisme de Toland est-il bien encore le christianisme? Pour Toland, on peut dire qu'il y a un christianisme authentique, mais à jamais perdu, et dont il ne subsiste que de multiples caricatures. Ne méritent, dit-il, le nom de « chrétiens », ni ceux qui défendent les préjugés et pratiques idolâtres (relatives, par exemple, au culte des morts et à l'immortalité de l'âme), c'est-à-dire défendent ce que Jésus-Christ était venu détruire ${ }^{52}$; ni ceux qui tiennent le christianisme pour une faction politique, alors que c'est une institution destinée à rectifier les moeurs et à donner des idées justes de la divinité ${ }^{53}$.

On ne peut toutefois clarifier cette thèse de l'existence d'un christianisme originel perdu, que par le détour des textes où Toland montre que, à travers les siècles, les divers visages du christianisme ne sont que les multiples déformations imposées par l'imposture cléricale. La conception de l'herméneutique biblique de Toland devient ici essentielle, et la comparaison avec celle de Locke peut s'avérer fructueuse.

Dans la Préface de Reasonableness of Christianity, Locke fait remarquer que c'est la divergence observée entre les doctrines des théologiens sur le sens à donner à la chute d'Adam qui l'avait conduit à un réexamen attentif du texte même des Écritures. Au demeurant, le titre même de l'ouvrage donne à voir une certaine hardiesse. Locke propose, en effet, avec Reasonableness of Christianity, as delivered in the Scriptures, de se fier à la

50. Ibid., \& 18 : «Nothing can be said a mystery because we are ignorant of its real essence, since it is no more knowable in one thing than in another, and is never conceived or included in the ideas we have of things, or the names we give them.»

51. Ibid.

52. Letters to Serena, op. cit. supra n. 41, Letter III, « The Origin of Idolatry and Reasons of Heathenism », $\$ 21$.

53. Ibid. : "How little right these have to the denomination of Christians, who defend the very things which Jesus Christ went about to destroy, is evident to all them who do not consider Christianity as a politic faction or bare sound; but as an institution designed to rectify our morals, to give us just ideas of the divinity. " 
seule matérialité du texte et de libérer ainsi le lecteur de l'autorité des théologiens. La méthode d'interprétation de l'Écriture, à partir de l'Écriture seule, semble ici quelque peu inspirée des principes posés par Spinoza dans le chapitre vII du Traité des autorités théologiques et politiques. Est-ce à dire que Locke irait jusqu'à traiter, comme le propose Spinoza, le texte des Écritures comme un objet naturel? Non, sans doute. Il n'est pas indifférent que, dans l'enquête proposée par Locke, les Écritures se ramènent essentiellement aux Actes des apôtres et aux Épîtres de saint Paul, des écrits qui, mieux que d'autres, fournissaient les matériaux propres à justifier le mystère du Christ comme Messie.

Avec cohérence, Locke se sert des résultats fournis par l'Essai, livre III, pour montrer que l'imperfection et les abus de langage sont comme les causes naturelles de l'obscurité des textes, la domination cléricale n'en étant qu'un effet. La Préface aux Commentaires des Épîtres de saint Paul est ici un document du plus grand intérêt, car elle fournit, à elle seule, le petit traité d'herméneutique de Locke.

Selon un singulier contraste avec Toland, c'est la parole de saint Paul qui, pour Locke, mérite, plus que tout autre, d'être interprétée, en tant que véhicule direct de la parole de Dieu ${ }^{54}$. Pour le faire, Locke énumère toutes les difficultés, d'ordre interne et externe, qui sont autant d'obstacles à la compréhension du message de l'apôtre. Les difficultés internes sont de trois ordres :

1) La forme épistolaire crée une première cause d'opacité, car elle présuppose une connaissance des rapports entre saint Paul et son correspondant, connaissance qui rendait superflue l'explicitation par Paul du détail des circonstances des Épîtres, mais qui fait défaut au lecteur du xvil siè$\mathrm{cle}^{55}$.

2) Une autre difficulté, commune à toute la littérature néotestamentaire, est évoquée : le langage des Épîtres est le grec, mais Paul ne maîtrisait pas correctement la langue grecque (langue d'un peuple spirituel, mais frivole, note Locke), et l'écrivait donc à travers les idiomes hébraïques ou syriaques, qui lui étaient les plus familiers. D'où suit, pour la langue des Épîtres, une idiosyncrasie parfois difficile à interpréter ${ }^{56}$.

3) À ces difficultés communes à tous les textes du Nouveau Testament, Locke en ajoute une autre, induite, selon lui, par le caractère et le style propres de Paul : celui-ci, « homme d'esprit vif et d'un tempérament fougueux ${ }^{57}$, suit les cheminements d'une pensée riche et vagabonde, qui se

54. M. C. PrtassI, art. cit. supra n. 3, « Saint Paul retrouvé», p. 41-54.

55. Commentaires des Épitres de saint Paul (cité par la suite comme CSP), Works, p. IV.

56. CSP, p. IV-V.

57. Ibid. : " He was, as is visible, a man of quick thought, and warm temper, mighty well versed in the writings of the old testament, and full of the doctrine of the new. All this put together, suggested matter to him in abundance, on those subjects which came in his way. " 
plaît aux digressions et aux parenthèses ${ }^{58}$; change de sujet ou d'interlocuteur en cours de route ${ }^{59}$; ou bien entreprend, contre toute attente, de répondre à une objection ${ }^{60}$.

Ces obstacles d'ordre interne sont renforcés par des obstacles externes qui, pour externes qu'ils soient, n'entravent pas moins une claire intelligence du texte paulinien. Ainsi :

4) Locke critique avec une énergie particulière la division, déjà très contestée, du texte des Épîtres en chapitres et en versets ${ }^{61}$, division artificielle introduite dans le texte par Robert Estienne, lors de son édition du Nouveau Testament. Cette division est, selon Locke, à l'origine de toutes les lectures dogmatiques du texte; elle a favorisé la transformation de passages obscurs en aphorismes soi-disant clairs, dûment réinterprétés par diverses orthodoxies contradictoires ${ }^{62}$.

5) Enfin, très sensible à l'incommunicabilité des individus entre eux, et des langues entre elles ${ }^{63}$, Locke fait remarquer que les traductions " reconnues » des Épîtres sont, depuis des siècles, imbibées par les tournures propres à la langue de traduction. Ainsi, toute compréhension du texte sacré se constitue à partir de la culture propre du lecteur; celui-ci comprend plutôt ce qu'il aurait dit lui-même, que ce que l'apôtre voulait dire $^{64}$.

Pour pallier ces multiples difficultés, Locke propose des principes herméneutiques qui, de Érasme à Spinoza, tendaient à devenir traditionnels. Tout d'abord, une lecture d'ensemble ininterrompue du texte; ensuite, une attention au dessein général de l'apôtre; enfin, une comparaison de textes

58. Ibid. : «In this posture of mind, it was impossible for him to keep that slow pace, and observe minutely that order and method of ranging all he said, from which results an easy and obvious perspicuity. To this plenty and vehemence of his, may be imputed those many large parentheses which a careful reader may observe in his epistles. "

59. CSP, p. VI : "The frequent changing of the personage he speaks in, renders the sense very uncertain, and is apt to mislead one that has not some clue to guide him; sometimes by the pronoun I, he means himself, sometimes any christian, sometimes a Jew; and sometimes any man. "

60. Ibid.

61. CSP, p. VII : « [The first difficulty is] the dividing of them into chapters and verses as we have done; whereby they are so chopped and minced, and, as they are now printed, stand so broken and divided, that not only common people take the verses usually for distinct aphorisms, but even men of more advanced knowledge, in reading them, lose very much of the force of the coherence, and the light that depend on it. 》

62. CSP, p. IX : «If the quotation in the verse produced were considered as a part of a continued coherent discourse, and so its sense were limited by the tenour of the context, most of these forward and warm disputants would be quite stripped of those, which they doubt not now call spiritual weapons. »

63. Essay, III, chap. IX, sect. 22.

64. CSP, p. X : «St Paul's Epistles, as translated in our English Bible, are now, by long and constant use, become a part of English language, and common phraseology, especially in matters of religion : this, every one uses familiarly and thinks he understands. " 
pauliniens entre eux ${ }^{65}$. Sur un mode en apparence spinoziste encore, Locke souligne qu'il faut comprendre les Épîtres de saint Paul, par le contexte des seules Épîtres ${ }^{66}$; alors il considère les Épîtres comme un texte parmi d'autres, soumis aux mêmes impératifs d'interprétation. Mais il retourne à la conception liminaire des Épîtres comme récit, lorsqu'il s'agit de fonder tous les dires de saint Paul sur l'inspiration initiale fournie par Dieu sur le chemin de Damas ${ }^{67}$. Accrochée à un tel mystère, comme aussi à une conception subjectiviste de la conscience impartiale du lecteur face au texte ${ }^{68}$, l'herméneutique lockienne reste donc une herméneutique de transition $^{69}$, et ne parvient pas à traiter le texte de Paul comme un document ${ }^{70}$. C'est ce que fera Toland, au prix d'un renversement total de l'autorité de l'apôtre. En sorte que, là où Locke fait, en matière d'herméneutique biblique, l'exposé d'un philosophe croyant, soucieux de défendre les droits de la conscience individuelle, Toland adopte le point de vue très différent d'un anthropologue.

Le Nazaréen (augmenté du Manuscrit irlandais des quatre Évangiles) en fournit la meilleure preuve. L'ouvrage a sa source dans la découverte et l'analyse de deux manuscrits qui, similaires à certains textes apocryphes, et confrontés aux textes reconnus comme « sacrés », tendaient à subvertir les idées fausses et pernicieuses qui, selon Toland, masquaient le christianisme originel $^{71}$. Dans une telle entreprise, ce n'est plus la défense du christianisme originel comme "vraie religion » qui est en cause, mais la mise à jour de la fonction idéologique de toute religion.

Fondamentalement, la « découverte », que Toland s'attribue du manuscrit de l'Évangile de Barnabé ${ }^{72}$, lui permet de mettre à jour la précarité de tous les témoignages et documents relatifs à l'histoire humaine. En effet, " parmi le grand nombre d'Évangiles, d'actes et de révélations qui se trouvent répandus dans les premiers temps de l'Église ${ }^{73}$, et que la majorité des chrétiens ont rejetés comme apocryphes, il en reste quelques-uns qui subsistent, soit en entier (comme l'Évangile de Jacques), soit sous

65. CSP, p. XIII-XXIII.

66. CSP, p. XVI : "All this, and a great deal more, necessary to guide us into the true meaning of the epistles, is to be had only from the epistles themselves."

67. CSP, p. XV : "I remembered that St Paul was miraculously called to the ministry of the Gospel, and declared to be a choosen vessel; that he had the whole doctrine of the gospel from God immediate revelation. "

68. CSP, p. XXII-XXIII.

69. M. C. PTtassi, art. cit. supra n. 3, p. 52-54.

70. Sur la distinction entre l'Evangile comme récit, texte ou document, voir Pierre-François MOREAu, "La méthode d'interprétation de l'Écriture sainte. Déterminations et limites ", in Spinoza, science et religion, éd. Renée Bouveresse, Paris, Vrin, 1988, p. 109-113.

71. J. Champion, op. cit. supra n. 36, p. 194-224 et conclusion, p. 228-235.

72. Le Nazaréen, Lettre I, chap. v; sur l'histoire de la découverte de ce manuscrit, voir J. Champion, op. cit. supra n. 36, p. 125 et notes.

73. Le Nazaréen, chap. II, p. 12. 
forme de fragments, comme ce fut le cas pour l'Évangile attribué à Barnabé. Toland est convaincu d'avoir retrouvé cet Évangile; et il l'identifie avec ce qu'il appelle l'Évangile des Mahométans ${ }^{74}$.

L'objectif de Toland est de démontrer que « les différentes sectes du christianisme », ne sont, en fait, qu'une unique religion fondamentale et universelle. Il remarque que les Mahométans reconnaissent eux-mêmes «qu'il parut sur la terre six personnages éminents, Adam, Noé, Abraham, Moïse, Jésus et enfin Mahomet »; chacun ayant apporté une nouvelle institution religieuse qui lui semblait l'emporter sur les précédentes, « mais qui ne contenait néanmoins qu'une seule et même religion ${ }^{75}$. Pour atteindre cet objectif, en se servant des Nazaréens ou Ébionites comme porteparole $^{76}$, Toland invite son lecteur à mettre en doute l'autorité prise par Paul et par les Actes des apôtres, dans le cadre historique d'un conflit avec les autres apôtres (en particulier avec Pierre), sur le contenu de la loi pour les premiers chrétiens. De son temps, Paul a été perçu comme un impos-

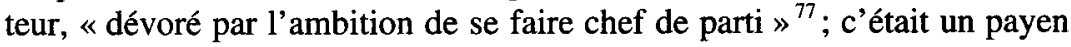
dont la conversion à la loi de Moïse n'avait obéi qu'à l'opportunisme le plus banal ${ }^{78}$. Quant à sa conversion ultérieure au christianisme, aucun témoignage ne rapporte quoi que ce soit de la révélation reçue sur le chemin de Damas; on peut donc penser, suggère Toland, que les Actes des apôtres ne sont que «l'histoire de Paul » ${ }^{79}$.

À partir de ces « données », Toland montre que c'est Paul de Tarse qui est à la racine du schisme entre ceux qu'il propose d'appeler, d'une part, les « Chrétiens-Juifs » et, d'autre part, les «Chrétiens-Gentils ». C'est Paul qui a inventé le dogme selon lequel Jésus-Christ était venu abolir la loi de Moïse $^{80}$. À cent lieues de cela, les premiers Chrétiens ou «ChrétiensJuifs », percevaient Jésus comme l'un des leurs, désireux non de détruire la loi sous laquelle il était né, mais seulement de la parfaire; les Juifs n'étaient donc nullement libérés des obligations de la loi. Seuls les

74. Ibid., chap. III, p. 17 : « Après ce que je viens de dire de l'Évangile de Barnabé, j'en viens présentement à l'Évangile des Mahométans, qui est probablement, dans sa plus grande partie, le même que celui de Barnabé, lequel n'est par conséquent pas si absolument perdu que se le sont persuadés tous les auteurs chrétiens. 》

75. Ibid., p. 18.

76. Ibid., chap. Ix, p. 48 : Toland fait remarquer que l'appellation d'Ébionites, ou « les gueux », avait été donnée aux Nazaréens comme une marque de mépris; et il ajoute que, encore aujourd'hui, on appelait aussi Ébionites les premiers Protestants en Flandre.

77. Ibid., chap. X, p. 58 .

78. Ibid., chap. XI, p. 64 : Toland « rapporte » que, lors d'un séjour à Jérusalem, Paul avait été pris du dessein d'épouser la fille du grand prêtre; c'est à cette fin, qu'il se fit circoncire. Comme elle le repoussa, il fut pris d' « un dépit violent, qui le porta à écrire contre la circoncision, contre le Sabbat et contre l'observation de la loi mosaïque ».

79. Ibid., p. 63.

80. Le Nazaréen, chap. viII et $\mathrm{x}$ : les Nazaréens (ou Ébionites) «rejetaient les Épîtres de Paul comme celles d'un ennemi et d'un imposteur ». 
«Chrétiens-Gentils» y étaient d'emblée soustraits; ils n'étaient, note malicieusement Toland, soumis qu'à «quatre préceptes »- préceptes relatifs aux rites extérieurs et si analogues à la loi de Moïse, qu'ils permettaient à tous de se réunir dans l'amitié d'un repas partagé ${ }^{81}$. Mais le contenu universel de l'enseignement du Christ (qui était présent chez les Juifs, chez les Gentils et les Mahométans, comme « Christ intérieur » ${ }^{82}$ ), restait indépendant de la fonction politique de la loi des Hébreux ; ces derniers devaient donc persévérer dans l'obéissance aux prescriptions de Moïse, qui demeurait la condition de leur cohésion ${ }^{83}$. C'est, observe Toland, l'importance donnée ultérieurement par les théologiens aux Épîtres de Paul qui a fait totalement oublier que "pendant un certain temps » les premiers Chrétiens étaient tous des Juifs ${ }^{84}$. Et ce n'est pas le moindre mérite des Nazaréens que d'avoir regardé Paul comme un apostat, un transgresseur de la loi, qui «s'était intrus dans le vrai christianisme ", comme un étranger à la doctrine de Jésus, à laquelle il avait substitué ses propres "révélations " ${ }^{85}$.

Par ailleurs, le manuscrit irlandais des quatre Évangiles fournissait une sorte d'utopie rétrospective de ce que Toland semble avoir considéré comme le christianisme authentique. Si l'on met en évidence que la religion des anciens Irlandais peut se confondre avec celle des Écossais, à la façon dont on peut parler d'un christianisme des Juifs, un autre des Gentils et un autre des Mahométans, c'est qu'il y a, dans ces institutions humaines, un fond de sagesse et de vérité qui n'est attaché à aucun territoire ${ }^{86}$. Certes, les faits et la nature craintive des hommes attestent que la superstition tend toujours à faire des progrès dans toute société, et qu'elle est continûment encouragée par les prêtres, cultivant l'ignorance des hommes à leur pro-

81. Ibid., chap. xI, p. 78-79 : l'apôtre Jacques avait écrit aux Gentils qui avaient reconnu Dieu « de s'abstenir seulement de la pollution des Idoles, de la fornication, des chairs étouffées et de sang ». Toland précise que, sans ces quatre préceptes, "il ne saurait y avoir de communion entre eux et les Chrétiens-Juifs ». Car, ajoute-t-il fort joliment, « il ne peut jamais y avoir de société cordiale entre des gens qui ne peuvent jamais boire et manger ensemble ".

82. Ibid., chap. XvII, p. 122-124 : Toland explique qu'il y a une loi de nature, que Jésus a observée à la perfection, que Moïse ne pouvait manquer de connaître et que Cicéron exalte dans ses écrits; loi d'amour, de joie, de paix et de patience, sans laquelle aucune société ne serait possible. Toland, in ibid., chap. XIv, p. 95-97, juge d'Epiphanius, le plus partial des théologiens, « qu'il n'aurait pas dû estimer [les Juifs] moins bons chrétiens par l'homme intérieur, parce qu'ils étaient Juifs par l'homme extérieur ».

83. Le Nazaréen, chap. xII, p. 80 : « Nous ne voyons rien dans le décret des Apôtres qui ordonne aux Juifs-Chrétiens de quitter leur ancienne loi, ni qui permette aux Chrétiens-Gentils de se soustraire aux quatre préceptes qui leur sont imposés. »

84. lbid., p. 47.

85. Ibid., p. 52-53; Toland ajoute, in ibid., p. 58 : « On reconnaît par sa propre déclaration [de Paul] qu'il voulait l'emporter sur Pierre et sur Jacques, et qu'il était dévoré de l'ambition de se faire chef de parti. »

86. Manuscrit irlandais, op. cit. supra n. 38, p. 260 : «La vérité ne se renferma pas dans les bornes d'un certain pays, et la raison n'est pas la production d'un terrain à l'exclusion des autres : l'une et l'autre sont toujours les mêmes dans tous les pays du monde.» 
$\mathrm{fit}^{87}$. C'est pourquoi il est du devoir de « l'historien » de mettre à jour l'origine des superstitions, de les expliquer et, en définitive, de les faire disparaître par la connaissance qu'on en prendra.

Le christianisme se confond dès lors avec une religion morale universelle, très simple dans ses prescriptions, au nom de laquelle Toland accepte de se dire lui-même « Nazaréen ${ }^{88}$, du nom des seuls véritables premiers chrétiens. Il ne prône toutefois pas l'uniformité d'une religion qui n'aurait de catholique que le nom. Ce serait, dit-il, nier aveuglément la diversité comme si, sous prétexte qu'il y a une espèce humaine, on refusait de voir la réalité du partage entre les deux sexes ${ }^{89}$. Il ne serait pas souhaitable, dit-il «qu'il n'y eût qu'une seule communion de Chrétiens, parce que c'est une chose impossible dans la nature », et que ce n'est pas ce que veut dire le terme de communion des saints; je souhaiterais seulement, dit-il encore, qu'il ne se trouvât pas un seul homme dans aucune des différentes sectes du christianisme, qui pût croire que sa secte était la seule bonne et la seule recevable ${ }^{90}$. Qu'y a-t-il, en effet, de plus contraire au christianisme vrai, que ce christianisme payen par lequel on se damne les uns les autres? Et par lequel on rivalise en célébrations ridicules, sans rapport avec l'enseignement de Jésus-Christ ${ }^{91}$ ? Il est affligeant de constater que :

« chaque secte et chacune de ses branches a toujours prétendu être la seule vraie Église de Jésus-Christ, à l'exclusion de toutes les autres [... et que ce soit] encore la même chose aujourd'hui, entre tous les Protestans d'un côté et les Papistes de l'autre, sans parler des Grecs; chacun d'eux se glorifiant d'avoir par devers soi je ne sais quelle tradition et succession non interrompue, [est] la prétention la plus chimérique qui puisse se trouver dans la nature ${ }^{92}$.

Allant jusqu'au bout des réflexions sur l'intolérance et sur la religion authentique, Toland se représente comme Chrétien lui-même, à la manière dont l'étaient les Nazaréens ${ }^{93}$; aussi va-t-il jusqu'à dire (car tel était le « vrai système » des Nazaréens) que le Christ était un être humain exemplaire, et non pas quelque «Fils de Dieu ${ }^{94}$.

87. Letters to Serena, op. cit. supra n. 41, Letter III, « The Origin of Idolatry and Reasons of Heathenism ».

88. Le Nazaréen, chap. xvII-xvII : Toland y expose avec emphase son " système Nazaréen », qui réunit tous les croyants en la reconnaissance d'un seul Dieu; et il exprime, p. 135, sa répugnance à l'embrigadement comme suit : «Il y a longtemps que j'ai déclaré que je n'aimais pas les noms que les hommes se donnent les uns aux autres par rapport a la religion [...] J'annonce que celui de Nazaréen est celui que j'adopte pour plus d'une raison. »

89. Le Nazaréen, Préf.

90. Ibid.

91. Le Nazaréen, chap. xviI, p. 132-133.

92. Le Nazaréen, chap. $\mathrm{xx}, \mathrm{p} .151-153$.

93. Voir supra n. 88.

94. Le Nazaréen, chap. $\mathrm{x}$, p. 50-51 : « [La plupart des auteurs connus sous le nom de Pères] sont d'accord en ce point que les Nazaréens et les Ébionites soutenaient que Jésus- 
Comme Spinoza le rappelait dans le $T T P$, en hébreu, on rapporte métaphoriquement à Dieu, tout ce qui dépasse les normes habituelles du comportement, tout ce qui crée la surprise, voire l'émerveillement ${ }^{95}$; alors, la figure morale que représente Jésus suffisait, par elle-même, à le dire venu «de Dieu». Locke lui-même, analysant les expressions "Fils de Dieu » et « Royaume de Dieu », telles qu'elles étaient employées par les Juifs, faisait remarquer qu'il s'agissait de formules chargées d'espérance, destinées à désigner le Messie et la venue du Messie ${ }^{96}$. Plus radicalement, Toland affirme que ce sont les prêtres qui ont exploité de telles expressions par lesquelles certains Hébreux témoignaient, dans le cadre de leurs idiomes familiers, leur admiration à l'endroit du Christ. Mais Jésus-Christ n'a pas même la primeur du message qu'on lui attribue; car si les auteurs chrétiens avaient lu Cicéron, note Toland, ils auraient su que, dans toutes les sociétés humaines, on tend à appeler «Dieu», tout ce qui suscite l'admiration; alors ils se seraient épargné la peine d'écrire eux-mêmes ${ }^{97}$; c'est-à-dire de s'engager dans des controverses sans terme, où les sophismes ne le cèdent qu'à l'aveuglement.

Si la défense de la liberté d'expression, associée à un anti-papisme déclaré, produit une ressemblance de surface entre Locke et Toland, la conception ultra-individualiste de la conscience du croyant et de l'exégèse font de Locke un humaniste encore traditionnel. On peut dire que, pour Locke, la « vraie religion » assure le salut des âmes et, dans l'ordre social, aurait pour bénéfice secondaire de protéger l'individu. En complète rupture avec Locke, Toland fait, sur la divinité, des déclarations qui témoignent d'un agnosticisme foncier, qui fut tenu par beaucoup pour de l'athéisme ${ }^{98}$. Si donc les dieux ne s'occupent pas des affaires des hommes, on ne peut plus en appeler, comme le fait Locke, à une «vraie religion».

Christ n'avait été qu'un homme comme les autres; tant du côté de son père que de celui de sa mère; qu'il était fils de Joseph et de Marie; qu'il avait été juste, sage, excellent par-dessus les autres hommes, ayant mérité particulièrement d'être appelé Fils de Dieu, à cause de la vie très vertueuse qu'il a menée et des vertus extraordinaires dont il était doué. »

95. Dans le chapitre I du TTP, SpINOZa énumère les motifs qui, dans la langue hébraïque, conduisaient très couramment à attribuer une chose à Dieu; tout ce qui semble insolite, dans la Nature, pouvait être appelé « ceuvre de Dieu».

96. $R C$, Works, VI, p. 33.

97. Letters to Serena, op. cit. supra n. 41, Letter III, p. 118; dans Le Nazaréen, p. 122-123, Toland cite Cicéron, De Rep., liv. 3 , pour corroborer ce point, dont il s'attache à faire une opinion courante chez ceux qui ont un tant soit peu philosophé : «La droite raison est une vraie loi, convenable à la nature répandue parmi tous les hommes [...]. Elle n'a besoin ni d'interprétation, ni d'explication de qui que ce soit que d'elle-même; sa loi n'est pas autre à Rome qu'à Athènes; et elle n'est pas différente en un temps qu'elle a été dans un autre. »

98. Voir D. Berman, op. cit. supra n. 1. 
Dans une visée désormais très laïque, étrangère à l'auteur de l'Essai, le modèle d'un christianisme originel permet à Toland de justifier l'existence d'une Église d'État qui pouvait, à ses yeux, contrevenir aux fanatismes et fournir un instrument efficace de cohésion sociale ${ }^{99}$.

Geneviève BRYKMAN (juin 1994).

99. J. Champion, op. cit. supra n. 36, p. 218-222. 